\title{
Punjab Healthcare Commission's Regulation of General Practitioners of Allopathic Medicine
}

\author{
Ain-ul-Momina, ${ }^{1}$ Sumair Anwar, ${ }^{2}$ Ehsanullah Tarin ${ }^{3}$
}

\begin{abstract}
Background: Ever since inception, allopathic medicine has increasingly been practiced all over the world including Pakistan. However, a significant proportion of practitioners are unqualified quacks. In order to curb quackery and assure the provision of quality healthcare by clinics and other healthcare establishments the Punjab Government established Punjab Healthcare Commission. How far this body has achieved its objectives?
\end{abstract}

Methods: A qualitative study was conducted for responding to the research question. Participants were drawn purposefully representing stakeholders, including general practitioners of allopathic medicine, association of general practitioners, academy of family physicians, and Punjab Healthcare Commission. Interviews held were audio-recorded and transcribed verbatim, followed by coding and generation of themes.

Results: The study yielded themes: protection of the

Conflict of Interest: No

Funding Source: No

Momina A.U. ${ }^{1}$

Assistant Professor of Community Medicine

King Edward Medical University, Lahore

Anwar S. ${ }^{2}$

Associate Professor of Community Medicine

Shalamar Medical and Dental College, Lahore

Tarin E. ${ }^{3}$

Adjunct Faculty, University of Health Sciences

Assistant Professor, University of the Punjab rights of patients and practitioners; vague registration process; stakeholders' (mis) understanding that PHC was using registration and licensing as a mean for revenue generation; regulating general practitioners of allopathic medicine was promoting quackery; and that under the circumstances achieving quality in healthcare was a farfetched idea.

Conclusion: It is early to gauge the impact of PHC, which has a gigantic agenda. Yet, while there are signs it is achieving objectives, there is a need for PHC to improve its communication strategy and broaden stakeholders' base. Furthermore, while defining regulatory strategies, socioeconomic inequalities should be factored in. Since, without this being considered, no systemic level reform will be sustainable, yielding optimum results and paying dividends for a longer timeframe.

Key Words: General Practitioners, Regulation, Litigation, Quality of Care. Health Service Delivery.

\section{Introduction}

Ever since its inception, allopathic medicine has increasingly been a strong component of health systems all over the world; and is now a leading modality of treatment. ${ }^{1,2}$ Pakistan is no exception, and has a sizeable number of allopathic practitioners serving the population. ${ }^{3,4}$ But, due to the wide acceptance and associated financial benefits, a significant number of unqualified healthcare providers also pretend to be the allopathic practitioners. This complicity in malpractice often results in medical negligence ${ }^{5}$ and such incidents often get into the media, bringing the issue on policy agenda. ${ }^{6}$

Access to proper healthcare is people's right under 
Article 9 of the Constitution of Pakistan. Being conscious that the healthcare provision by the unauthorized medical practitioners amount to violating the rights conferred by the Constitution, the Punjab Government established a Punjab Healthcare Commission (PHC) as an autonomous body under PHC Act (XVI) $2010 .^{7}$ The aim is to "improve the quality of healthcare services and ban quackery in Punjab in all its forms and manifestations'.

This paper examines how far PHC has been successful in getting to its aim; and for that the strengths and weaknesses of the initiative are explored. The discussion extends to identifying the way forward in overcoming the barriers and improving health services delivery for the people. The paper concludes by underlining the importance of an effective communication strategy and fostering the partnership between PHC and the stakeholders.

In Pakistan, in addition to the allopathic, a range of traditional and alternative medicine practiced includes Ayurveda, Homeopathy, Unani, and Chinese. Furthermore, while the spiritual healers practicing mindbody medicine advice meditation, prayer, and mental healing, there are others prescribing natural therapies, like foods, dietary supplements, and herbal products. Massaging, bone settling, bloodletting and advising energy medicine are also practiced. ${ }^{8}$

In order to regulate traditional and alternative medicine in Pakistan, "Unani, Ayurvedic and Homeopathic Practitioners' Act of 1965" was promulgated. Under this Act, a Board of Unani and Ayurvedic System of Medicine is established. It is responsible, interalia, to register qualified persons, maintain adequate standards of practice, recognize training institutions, conduct research etc. The said Act prohibits Tabibs (practitioners of Unani medicine) and Vaids (practitioners of ayurvedic medicine) from using the title "Doctor". Likewise, Ordinance 65 of 7 June 1962, which is aimed to prevent "misuse of the allopathic system", allows only the registered (allopathic) medical practitioners to use the title "Doctor", perform surgery, and prescribe antibiotics. " Likewise, Pakistan Medical and Dental Council Ordinance 1962, amended in 2012 seek to establish uniform minimum standards of basic and higher qualification in medicine and dentistry. According to the said law persons holding valid registration are eligible to practice allopathic medicine.

However, despite these regulatory mechanisms, quacks continue to operate in Pakistan. A "quack" is "a person who pretends, professionally or publicly, to have the skill, knowledge, or qualifications, which in fact he or she does not possess". ${ }^{10}$ According to Pakistan Medical Association (PMA), the number of quacks in Pakistan exceeds 600,000, with some 70,000 operating in Karachi alone. ${ }^{11}$ These pretenders practice allopathic as well as traditional and alternative medicine, not infrequently with untoward consequences, evoking public uproar. Government authorities in the wake of such incidences, take measures, albeit often unstructured and ad-hoc, with doubtful results. ${ }^{11}$

In recent past the death of a 3 - year old girl Imanae Malik, allegedly due to negligence and malpractice in a local private hospital, drew the attention of civil society and judiciary. ${ }^{12}$ The response of Punjab Government was structured and reformative. A Punjab Healthcare Commission established under PHC Act (XVI) 2010 aim is to improve quality, safety and efficiency of healthcare delivery by the public and private healthcare establishments in Punjab province. Under the said Act, practitioners of allopathic medicine, often termed as General Practitioners (GPs) ${ }^{13}$ who are otherwise qualified and trained, but not registered with Pakistan Medical and Dental Council (PMDC), are considered quacks.

It has been almost five years since the PHC took off, but to what results? To respond to this question, however to date no empirical study is undertaken. This study fills this gap by objectively reviewing the acceptability and efficacy of the promulgated regulatory mechanisms; and to assist this enquiry a conceptual framework is defined.

In order to understand the regulatory mechanisms instituted by PHC a conceptual framework (Fig. 1) is defined drawing on the earlier work of T. Marmor and C. Wendt (2012). ${ }^{14}$ Accordingly, the GPs practicing allopathic medicine, in case of malpractice, are likely to be subjected to 'Quackery and Litigation', invoking the social or political and institutional actors on the one hand and monetary implications on the other hand. The former, whereas obliges the GPs for monetary inputs, also requires the government to install effective regulatory mechanisms; like the Punjab Healthcare Commission in this case. With regulatory mechanisms acting as catalyst, the monetary inputs translated into health personnel and facilities gear into the process or health activities like diagnosis, treatment etc. The ultimate outcome of regulatory mechanism is the transformation of quackery and litigation into health outcomes, i.e. patient satisfaction and improved health status of the population. 


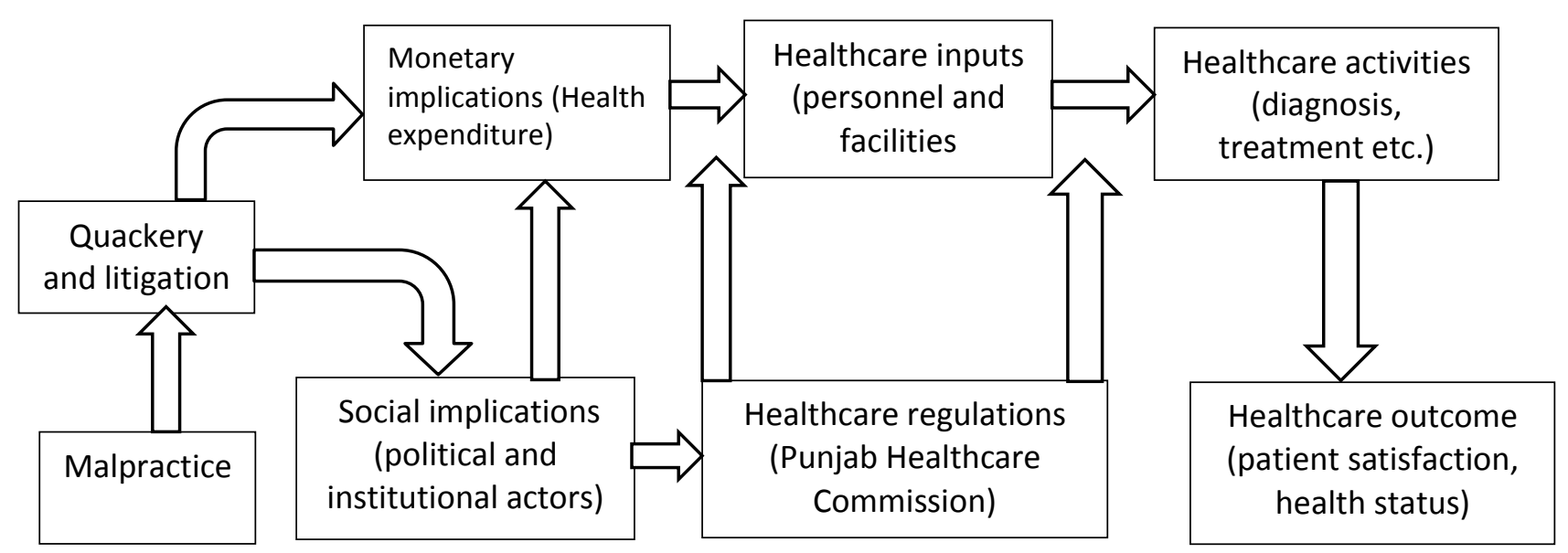

Fig. 1: Transformation of quackery and litigation to better health outcomes.

In this transformation phenomenon, the Punjab Healthcare Commission acts at two levels. Firstly, while the monetary inputs are translated into real inputs or healthcare inputs that are appropriate and according to standards; and secondly getting the process right, i.e. diagnosis and treatment of good quality. This study seeks insight into the relationship between GPs practicing allopathic medicine, who are agents in transforming inputs into processes (healthcare activities) and the PHC as regulator. The aim is to identify the strengths and weaknesses of the exercise of PHC in bringing allopathic medicine practitioners within its regulatory confines. The findings will help identify a way forward to help overcome barriers and consequently improve health service delivery for the masses.

Within the remits of this aim, the objectives of the study were:

1. To identify perspective held by general practitioners' of allopathic medicine about regulatory mechanism installed by Punjab Healthcare Commission;

2. To explore the strengths and weaknesses of the regulatory mechanisms as stipulated by the Punjab Healthcare Commission; and

3. Identify a way forward that can help in overcoming the barriers and consequently improve health service delivery for the masses.

\section{Methods}

A qualitative phenomenological design was used to explore the meanings and perceptions about $\mathrm{PHC}$ regulations in the manner those are implemented through in-depth semi-structured interviews. The participants were drawn purposefully from general practitioners of allopathic medicine (GPAMs), Association of General Practitioners (AGP), Pakistan Academy of Family Physicians, and PHC officials. Study was conducted in Lahore due to the presence of AGP and PHC offices here. The GPAMs running clinics independently, having MBBS qualifications recognized by PM\&DC and with over 10 years of experience were selected purposefully. The sample comprised four GPAMs, two senior officials one each from AGP and PHC. Quantitative data was collected through in-depth semi-structured interviews using a topic guide. An informed consent was sought and interviews held at participant's workplaces, each lasting for 45-55 minutes, were audio-recorded and transcribed verbatim. This data was substantiated with field notes. Ethical approval for the study was given by the Institutional Review Board of the Institute of Social and Cultural Sciences, University of the Punjab, Lahore.

Thematic analysis was done keeping interview as the unit of analysis. After immersion phase of determining relationship between GPAMs' practice and PHC regulation, initial codes were generated in a systematic fashion. Codes were used to create "big ideas" (or themes) from each interview. Descriptive analysis including collation of related data to each code followed by 'cut and paste' of data around each theme was performed. A subset of transcripts was also double coded by the members of the research group who had not earlier created codes. Disagreements and insights were discussed and alternative interpretations were incorpora- 
ted in the analysis. Finally, interview transcripts were indexed and mapped on the basis of recurring major themes with corresponding sub-themes. For the research design and analysis phase of study, the check on validity was that a topic guide was used to ensure a similar range of topics was discussed with each participant. Likewise, to ensure reliability two researchers read interviews separately and agreed on coding framework.

\section{Results}

The following themes that emerged from analysis are elaborated in the ensuing pages:

1. Protection of the rights of patients' and GPAMs,

2. Quality of healthcare offered by GPAMs.

3. Registration process invoked by PHC.

4. Licensing as a means to revenue generation by PHC.

5. Regulatory mechanisms sine-qua-non to promoting quackery.

\section{Protection of the rights of patients and allo- pathic practitioners}

Following its establishment the PHC has installed standardized procedure for lodging complaints and appeals, primarily to assure the rights of the patients as well as GPAMs are protected. This role of PHC is appreciated, as in past, study participants observed the investigation of complaints fully and formally was difficult and complex process. As a consequence, particularly patients were often nervous finding it difficult to lodge complaint and communicate their views about clinicians. Likewise, doctors didn't have any formal platform, which could protect their rights. A senior PHC official cognizant of the situation said, " $a$ two-way war was going on between patients and doctors and this left both parties, patients and doctors, vulnerable at the hands of a vague redress system."

In the absence of or due to the vague and complex complaints' procedure, patients would resort to lodging FIRs (First Information Report), seeking police intervention. In this scenario, the medical practitioners would feel threatened especially if the complainants were influential, as they could maneuver the outcome of police investigation. This was enunciated as, "in the past, influential complainants would lodge a case with Police and it would book the doctor under section 302 - intent to murder' (Senior PHC official). Likewise, a senior official of Pakistan Academy of Family
Physicians noted, "PHC is a protectorate for doctors. It has benefits!"

Although the role of $\mathrm{PHC}$ in protecting the rights of doctors has emerged, there is skepticism. PHC is bypassed and doctors are tried in the criminal courts, as was illustrated, "why are the doctors still being slapped with FIRs in the presence of PHC?" In such circumstances, a false case against a practitioner can harm reputation in community which s/he earned through years of hard work, possibly affecting adversely also his practice. The PHC in order to safeguard practitioners' rights should penalize complainants who had purposely vilified GPs, as otherwise as a senior official of Pakistan Academy of Family Physicians, questioned, "what is the net benefit to doctors (of PHC) if there is no mechanism to penalize a false accuser?"

\section{Quality in Healthcare}

Quality in healthcare is questioned, especially on account of the weak accountability mechanisms. In this regard, the study participants indicated, "the gap existed due to the standards and protocols (now defined by PHC) are poorly adhered to in healthcare delivery". Notwithstanding that however, the efforts of PHC in defining and enforcing standards for assuring quality in healthcare is recognized, like it was noted, "it's a good step, since before inception of PHC the GP and even the specialist practice was like an unbridled horse." Particularly, the participant added, "private practitioners would run their clinics with no care to monitoring quality in their practice".

One of the factors potentially contributing to poor quality healthcare is that till recently there was no legal requirement for GPAMs to enhance or maintain their knowledge and skills set. In many cases the GPAMs, after qualifying, would continue to practice even though their registration with PMDC had expired. In this manner patients are exposed to GPAMs who most likely had lost touch with the latest knowledge and potentially use obsolete treatment options. PHC therefore made it mandatory for clinics and other health establishments that before renewing registration they have assured Continued Medical Education of their clinical staff. This intervention was appreciated as, "Continued Medical Education, a requirement by PHC, was unheard of by GPs. But, it will be very helpful for them."

Lack of accountability in case of malpractice came to limelight during the proceedings of a famous case of "Imane Malik". Imane Malik was a young girl child 
who died at a private healthcare facility due to an IV injection. The case was heard at Supreme Court of Pakistan; it was found that there existed no system to bring to justice the accused in the private healthcare establishment. It was observed, "Imane Malik case's inquiry ordered by the Supreme Court of Pakistan revealed many lapses in the medical management that protocols were not followed". Further, while "in Public sector PEEDA (The Punjab Employees Efficiency, Discipline and Accountability) Act and certain other disciplinary measures exist, nothing of the sort is defined for the private sector. Instead of fixing their system, the private healthcare establishment, at best, would fire the doctor, but only if the complainant were influential persons.",

In order to assure quality, PHC laid down Minimum Service Delivery Standards (MSDS). Evidence suggests, those are not received well by GPAMs and their representatives. In this regard, the salient barriers identified were; i) lack of adequate human resource and physical infrastructure; ii) cost of implementing MSDS; iii) geographical disparities; and iv) lack of monitoring capacity.

\section{i) Human Resources and Physical Infrastructure}

PHC, as part of MSDS, requires the availability of specified number of trained human resources (HR). Pakistan is classified by WHO as a country that faces health workforce crisis (WHO, 2006). This situation when translated on ground, GPAM believed, "MSDS laid down by PHC require, e.g. hiring a qualified dispenser, certain number of janitors and a designated setup for minor surgical procedures. This requirement is unrealistic; and is opening a Pandora box!" GPAMs have invested their time and money in training their assistants and are confident in their capacity, despite they lack required credentials. They are rather wearisome of change (to get a new qualified assistant) and consider it a risk to their practice, as a senior official of Pakistan Academy of Family Physicians noted, "the assistants helping us at our practices have been trained by us over several years, we can't trust a new employee with our patients. We are responsible for our assistant's quality of service delivery."

\section{ii) Cost of Implementing Minimum Service Delivery Standards}

The GPAMs in Punjab largely cater to population with limited socioeconomic means and accordingly the capacity to pay. In such environment, hiring qualified ancillary staff and maintaining clinics according to MSDS requires not only investing more capital but also raising the overheads manifold. The GPAMs in order to keep their practice affordable for their clients charge nominal fees. Any increase in fee to adjust the cost, capital as well as operational, will not augur well for their practice. This was confirmed by a GPAM who expressed, "expecting high standards of care are impossible given the socioeconomic brackets of providers and purchasers."

\section{iii) Geographical Disparities}

There are geographical variations in terms of capacity both on the providers and purchasers side in the Punjab. That is, capacity whether of providers' or purchasers in Lahore or Rawalpindi cannot be equated with that in Bahawalpur or Rajanpur districts; and within these districts between urban and rural areas. But, PHC defined MSDS and made those applicable to all clinics and other health establishments irrespective of their location. That is, as a GPAM observed, PHC didn't take into account the disparities in the availability of resources that prevail due to the geographical positioning of GPAM's practice in Punjab. The ability to implement MSDS in a distant village in comparison to an upscale neighborhood of a large city is worth factoring in but was overlooked by PHC, as was expressed, "PHC shouldn't expect same standards of care to be followed at Chichoon ki Mallian as it does in Lahore."

\section{iv) Lack of Monitoring Capacity}

The PHC has installed system for monitoring the implementation of MSDS by clinics and other health establishments. Questions are raised about the capacity whether of the system per se or of those operating the system, including inter-alia the inspectors in PHC. In this regard, a senior official of Pakistan Academy of Family Physicians was concerned, "PHC simply does$n$ 't have the HR to inspect all healthcare establishments in the entire province." In their view, given that Punjab is a populous province with a large number of GPAMs operating all around and the limited number of PHC inspectors the entire objective of improving service delivery will fail.

\section{Unclear Registration Process}

The study revealed that GPAMs - the main stakeholders - found the registration process installed by $\mathrm{PHC}$ is complex and confusing. This was evident from 
the remarks: "You open a door to fulfill a PHC criterion and you find out that there is another door that needs to be opened and then another, the process is endless."(GPAM 2) Likewise, another GPAM noted, "the registration process is like quicksand once you register there is no way out, it seems like one is trapped." In a similar vein, a senior official of Pakistan Academy of Family Physicians noted, " $80-90 \%$ GPs up till now have no idea about the bylaws of PHC."

Pakistan Academy of Family Physicians in the wake of the aforementioned complexities blamed the authors of PHC rules and regulations for the voids enunciated here and there in this paper. The authors of PHC rules and regulations were expatriates with little knowledge about the indigenous service delivery structure. Also, the stakeholders involved were only from public sector, whereas the GPAMs comprise mainly the private healthcare. The design and development of PHC didn't have inputs from wider stakeholders, which led to this confusion and disconnect of GPAMs with output, i.e. rules and regulations. Expressing himself, a GPAM noted: "PHC rules and regulations were since made by international consultants who had little understanding of Pakistani realities are impractical", and "stakeholders consulted during formulation were practitioners from public sector, and the private sector was not consulted during the formation of PHC."

\section{Revenue Generation}

The PHC charges the clinics and other healthcare establishments for registration, licensing and processing renewals. However, the GPAMs and their representative casted serious doubts about PHC's monetary ambitions, an accusation categorically denied by a senior official in PHC, who said, "revenue generation is certainly not our aim or objective and we aren't making any profit of it. Our financial records are a testament of this." The PHC, as proclaimed by one of its officials, is not a profit making enterprise; instead it uses its own resources to facilitate operational activities.

Another lacuna identified is the level of fee levied. It is same irrespective of the sizes, whether it is a 50 bedded hospital or a $4-5$ bedded facility. Since the former would have more revenue compared to the latter, yet charged the same add to the misgivings of GPAMs. Many GPAMs have beds at their clinics for patients who need few hours of indoor care or are in need of IV infusions. On this account they feel their clinics shouldn't be categorized as hospitals and hence not charged accordingly. Therefore, Pakistan Academy of Family Physicians has strong reservations over fee structure, "a clinic with 2-3 beds has the same licensing fee as for a 50 bedded hospital. Ridiculous!"

Furthermore, the same amount is levied as fee regardless of the location of clinic or other healthcare establishment. It was a cause of concern, since many GPAMs have clinics in areas where population has limited capacity to pay. For clinics in areas which are financially unprofitable, the cost of registration was a burden as they already struggle for livelihood. Also, GPAMs are not told upfront total registration charges and whether this was a one off or recurring. As a result, they get roped in to paying year-after-year. In this regard, apprehensions of GPAMs were: "as one comes within their ambit, registration charges keep on increasing one step after the other." Furthermore, "this registration process is only designed for people born with a golden spoon in their mouth." That is, it is hard for an ordinary GPAM to pay PHC.

\section{Promotion of Quackery}

PHC was established inter-alia with the objective to curb quackery. The GPAMs and their representative body therefore feel that going after quacks would have been the priority for PHC. Instead it started with GPAMs bringing them in the fold of registration, whereas quacks continue to practice with liberty without any fear of their clinics being sealed or going behind bars. In view of GPAMs, this tantamount to promoting quackery, elicited as: "Quacks are practicing with impunity and the government is doing nothing to hold them accountable, even a medical store salesman is prescribing medicine and is not under the PHC radar at all." Likewise, another GPAM said, "Why PHC isn't getting rid of quacks first; it's like trying to clean water without removing sand particles from it."

In the current scenario quacks charge much less than GPAMs. Raise in GPAM's fee that is likely on account of registration charges and to fulfill MSDS will make patients seek quacks' advice, particularly in remote and marginalized areas. For example, a GPAM was explicit in his concerns for losing out his practice to quacks, "the cost incurred for registering with PHC will transfer to the patient. This will divert our clientage to quacks, who already charge less than us." Likewise, GPAMs feel that with PHC sealing GPAM's clinics and doing nothing about quack facilities will increase people's faith in quacks; thus eventually harming allopath practice. Fearful of this consequence, a senior official of Pakistan Academy of Family Physicians said, "if PHC seals our clinics, it will bring a 
very bad name to the allopathic practice. The quack down the lane will give people more reason to doubt allopathic practice."

\section{Discussion}

In developing countries such as Pakistan, for most people requiring healthcare the first point of access is private sector providers, mainly GPs. ${ }^{15}$ Therefore, they are an important focus for the governments which aspire to enhance the performance of their health system. ${ }^{15}$ As Wangombe argues, regulation of health care delivery is essential for assuring quality in healthcare, GPs should be brought within the folds of regulation. ${ }^{16}$ In this manner, while unqualified persons will not be able to practice, mushrooming of illegal clinics will be prevented and standards of care will be maintained. But, health regulation is a complex concept and, as Nunes identifies, while different regulatory modalities could be successfully used, independent regulatory agencies are valuable not only as tool for improving performance, but also as an instrument of assuring social rights. ${ }^{17}$ This concept is upheld by this study, as findings overwhelmingly support PHC as an independent healthcare regulatory authority that will assure the rights of the patients as well as GPs. In this regard, however Robuya claims that low literacy level of patients having little idea of individual rights, and contrarily higher social status enjoyed by the medical doctors is likely to limit the former in acquiring or/and exercising their rights. ${ }^{18}$ This deduction was also endorsed by the results of a study done in Turkey. It was found that only $9 \%$ of patients were aware of the regulation about patients' rights. ${ }^{19}$ Our study however did not take the patient's account, although it would be worthwhile for future studies to explore patients' awareness of their rights and in this regard, how PHC could benefit them. Like patients or healthcare recipients it is equally important to safeguard the rights of GPs. In this vein, Sheather observed that unwarranted criticism and penalization of doctors by people with little experience of practical and personal complexities of healthcare delivery can be damaging for the overall effectiveness of the healthcare system. ${ }^{20}$ In an editorial, Doyle explains that unjust accusations of professional misconduct and unreasonable demands by patients can stretch physicians' capacity for empathy and respect to breaking point. This situation puts enormous stress on doctors, ${ }^{21}$ and as Cozens found creates a complex professional environment compounded by alcohol abuse, along with depression and burn out, marital troubles and general lack of happiness with clinical work. ${ }^{22}$

Quite often patients perceive services by private healthcare providers attractive, while the evidence suggests otherwise, as Brugha in his seminal paper found serious deficits in the quality of services ${ }^{23}$. Therefore, Ugur argues for assuring quality standards and invoking health regulation that can overcome deficits in service management. ${ }^{24}$ The development and implementation of MSDS mandated to PHC is of immense significance, as it has strong potential for bringing in a substantial modicum of quality in service delivery at all levels. The Punjab Health Strategy 2012-20 implicitly states the role of PHC in the implementation of MSDS in private healthcare which includes GPs. Specifically, it advises:

- "Apply MSDS for private sector through Punjab Healthcare Commission and support its implementation through Punjab Health Foundation."

- "Develop guidelines on quality measures at provincial level and institute clinical audits and clinical assessments through Punjab Healthcare Commission."

- "Develop and implement standards for brining culture of accreditation in the province through support of National Standards Committee on Healthcare."

However, MSDS laid down by PHC are not well received as some of their requirements are not compatible with available human and physical resources. Similar concerns are also raised in a study conducted by Ramesh Bhatt in India. He observed that the growth of paramedical staff and their training hasn't kept pace with the increasing number of private healthcare facilities. Furthermore in his views, the shortage and high cost are the leading problems facing private providers in hiring trained staff for their clinics. In addition, the financial, social and geographical disparities need to be factored in while defining and implementing milestones for promulgating regulations. ${ }^{25}$ Fidler also found in his research on new international health regulation that quality of healthcare would always benefit from a sound regulatory system in more than one ways. Health regulation when devoid of the solid legal foundations is open to ill - conceived interpretation if not downright abuse. ${ }^{26}$ Before creation of PHC, alleged cases of medical negligence and malpractice would invariably end up with Police, having little or no expertise to precisely ascertain irregularities in medi- 
cal service delivery. Influential persons would therefore use their resources and many a times wrongfully implicated practitioners for medical negligence that actually didn't warrant harsh legal battles, vice versa the doctors also got scot free by wielding their influence.

Like in this study, a review of Kenya's Regulatory Framework for Private Healthcare Services found that the existing laws and statutes cover most areas of concern in the health sector (e.g. licensing of practitioners, premises, and sale of drugs). Nonetheless, there is a need for a strong capacity to monitor within the government and regulatory agencies, in order to improve the operations of the private healthcare providers. ${ }^{27}$ The review concludes that the legal restrictions are only successful if a well - resourced regulatory framework was in place as well as a functioning judicial system for enforcing and sanctioning. But, awareness of such arrangements was key as otherwise the entire exercise could be questionable. ${ }^{28}$ This was also the case for PHC, due to information deficit, ambiguities and the misconceptions. As observed earlier, therefore there is a need to publicize and disseminate information about the role of PHC amongst stakeholders. Without this, the broad based and onerous mandates of registration and licensing would create further ambiguities and generate ill - will for the PHC. The same information deficit is the cause célèbre of this notion that registration and licensing are instruments of revenue generation for the Provincial Government, which is not the reason for its creation. Like other developing countries Pakistan has introduced Healthcare regulation without however objectively assessing its efficacy. In fact, some similar evidence comes from other developed countries. For example, Walshe in his research paper questions the outcomes of regulation and is doubtful about the extent to which regulation has achieved its objectives in the US and UK. ${ }^{29}$ Given this uncertainty about the effectiveness of regulation, even in the developed world, it is all the more pertinent for the researchers to further explore the performance of PHC.

\section{Conclusion}

The formulation, promulgation and implementation of regulation of GPAM's practice is a mammoth task, and this initiative will not come to fruition if it stays a stand - alone effort of the PHC. It needs strong partnership with wider stakeholders, directly or indirectly involved in the task of regulating practice in allopathic medicine. In the same vein however, the role of orga- nizations representing GPAMs, inputs from individual GPAMs, and feedback from patients can be pivotal in identifying the gaps in PHC conducting its business and bridging them with practical solutions.

The mandate of PHC needs to be explained to all stakeholders. With them, the initiatives, successes and failures should be communicated and shared. Given the deficit in healthcare quality, unethical and illegal practices, health regulation are of highest priority, albeit with gradual implementation. Furthermore, while defining regulatory strategies, spatial and socioeconomic inequalities need to be consciously factored in. Since without this being considered, no systemic level reform will be sustainable, yielding optimum results and paying dividends for a longer timeframe.

\section{References}

1. Katherine E, Gundling. When Did I Become an "Allopath"? MD Arch Intern Med. 1998; 158 (20): 2185-86.

2. Dearden A R, Graham G I, John R B, Jones J S. Patient care and the general practitioner. BMJ. 1994; 309: 1144.

3. Pakistan Medical and Dental Council.Total Number of Doctors / Dental Surgeons/Specialists [Internet]. 2015 [cited 2015 Sep 8].

4. Al Junaid $\mathrm{S}$. The role of private medical practitioners and their interactions with public health services in Asian countries. Health Policy Plan. 1995; 10 (4): 33349.

5. Memon, S Amjad. Rural Surgery in Pakistan. Academic Journal, 2006; 30 (9): p1628.

6. Yusuf H. Mapping Digital Media. A Report by the Open Society Foundations [Internet]. Pakistan [cited 2013 Jun 21].

7. Punjab Healthcare Commission Act 2010. The Punjab Gazette (Extraordinary), p 465-77.

8. Hussain S, Malik F, Khalid N, Qayyum MA and Riaz H. Alternative and Traditional Medicines Systems in Pakistan: History, Regulation, Trends, Usefulness, Challenges, Prospects and Limitations A Compendium of Essays on Alternative Therapy, 2012 Jan] , ISBN: 978953-307-863-2.

9. Zhang X. editor. Legal Status of Traditional Medicine and Complementary Alternative Medicine: A Worldwide Review [Internet]. Geneva: World Health Organization; 2001 [cited 2015 Nov 26].

10. Quack [Internet]:Dictionary.com. [cited 2015 Nov 26].

11. Anti quackery drive [Internet]. Karachi: Health Department. [cited 2015 Nov 26].

12. Full story - Imanae Malik Killed by Doctors Hospital [Internet]. Lahore [cited 2016 Jan13].

13. General Medical Council. Working with Doctors Work- 
ing for Patients [Internet]. United Kingdom [cited 2015 Sep 8].

14. Marmor T, Wendt C. Conceptual frameworks for comparing healthcare politics and policy. Health Policy 107.2012: 11- 20.

15. The use of private-sector contracts for primary health care. WHO bulletin.

16. Wang'ombe JK, Mwaniki D, Mitula P, Mugo MG, Nzoya D, Muasya I. Capacity of Non-Governmental Providers in Delivery of Health Care in Kenya. Informing and reforming, 1998; (7-8): 2-6.

17. Nunes R, Brandão C, Rego G. Public Accountability and Sunshine Healthcare Regulation. Health Care Anal. 2011; 19 (4): 352-364. doi:10.1007/s10728-010-01566.

18 Khan RI. Informed consent and some of its problems in Pakistan. J Pak Med Assoc. 2008; 58 (2): 82-4.

19. Kuzu N, Ergin A, Zencir M. Patients' awareness of their rights in a developing country. Public Health, 2006; 120 (4): 290-296.

20. Sheather J. Health professionals and human rights campaigners: different cultures, shared goals. Postgrad Med J. 2009; 85:148-51. doi:10.1136/pgmj.2008.074799.

21. Doyal L, Doyal L, Sokol D. Good medicine, human rights and the rights of doctors. Postgrad Med J. 2009; 85: 113-14. doi:10.1136/pgmj.2008.077420.
22. Cozens JF. Doctors, their wellbeing, and their stress. BMJ 2003; 326.

23. Brugha R and Zwi A. Improving the Quality of Private Sector Delivery of Public Health services: challenges and strategies. Health Policy and Planning; 13 (2): $107-$ 20.

24 Unger JP, Marchal B and Green A. Quality standards for health care delivery and management in publicly oriented health services. Int J Health Plann Mgmt. 2003; 18: S79-S88.

25. Bhat R. Characteristics of Private Medical Practice in India: A Provider Perspective. Health Policy and Planning. 1999; 14 (1): 26-37.

26 Fidler DP and Gostin LO. The New International Health Regulations: An Historic Development for International Law and Public Health. The Journal of Law, Medicine and Ethics, 2006; 34 (1): 85-94.

27. Muthaka DI, Kimani DN, Mwaura S and Manda DK. A Review of the Regulatory Framework for Private Healthcare Services in Kenya. In. Social Sector Division Kenya Institute for Public Policy Research and Analysis. Discussion Paper No. 35. 2004 March.

28 Walshe K. Regulating Healthcare: A Prescription for Improvement? Open University Press: Philadelphia 2003. 